\title{
Underbalanced Drilling Optimum Methodology
}

\author{
Wei Zhang ${ }^{1}$, Wenhui Dang ${ }^{1}$, Wenbo Zhang ${ }^{1}$, Xiaorui Sun ${ }^{1}$ and Zhongxi Zhu ${ }^{2, *}$ \\ ${ }^{1}$ Engineering Technology Institute of PetroChina Xin Jiang Oil Field Company, 834000, Karamay, Xinjiang, China \\ ${ }^{2}$ Petroleum Engineering College, Yangtze University, 430100, Wuhan, Hubei, China
}

\begin{abstract}
A methodology to properly screen all UBD techniques to reduce failure/misapplication and align objectives with expectations had been absent. The paper addresses the latest enhancements to better understand and screen options for UBD operations according to two aspects: formation applicability and engineering applicability. The screening tool considers a range of economic and technical parameter to provide a relative ranking for each candidate of the Underbalanced drilling (UBD) technique. As a case, the methodology was available to optimize the UBD techniques in a risky exploration well in Xinjiang oilfield, which provided guidance and technical support to screen the UBD candidate, design the drilling modeconversion, and establish the emergency schedule.
\end{abstract}

\section{Introduction}

Underbalanced drilling technology is an adaptive drilling process that enables a more precise control of wellbore pressures through the use of rotary blowout preventer and engineered processes. The drilling process is becoming an important tool for drilling many oilfields with depleted reservoirs, narrow pressure windows, and/or drilling issues. Drilling improvements stem from mitigating common drilling hazards caused by lost circulation in weak zone, discovering early oil/gas reservoirs, decreasing adequately formation damage, shortening drilling time and cutting drilling costs, on both a well-to-well and field-to-field basis. The drilling processing can provide a variety of benefits during the drilling operation and production phase. However, UBD as a high investment to get high returns technology can fail to deliver full potential because unsuitable candidates are drilled or the inappropriate drilling technology is applied. An important aspect of UBD success is proper candidate screening, which can provide an understand of benefits and risks. Many research institutions and service companies already have studied to screening the UBD candidate1,2, and the formation pressure profile prediction as the key factor is focused on, but to properly apply UBD, other factors besides the pressure prediction should also be considered comprehensively to more fully evaluate the UBD applicability. An UBD candidate screening method is proposed according to two aspects: formation applicability and engineering applicability.

\section{Formation lithology and sensitivity}

Stabilized borehole depending on formation lithology such as carbonatite, limestone and high intensity sandstone, is a precondition for UBD. Formation lithology must have enough intensity and strength to maintain the borehole not to collapse. Formation sensitivity usually refers to the water sensitivity of clay. The water sensitivity increases with increasing the clay mineral content in the rock, such as montmorillonite, kaolinite, illite and chlorite. Montmorillonite has strong hydrophilicity and high cation exchange property which makes it have intense water sensitivity and expand the volume 6 to 10 times after water influx. The adhesive force of kaolinite more common in the reservoir between the lattices is weak, and easily converts into other clay minerals when water invades. So, comprehensive analysis and assessment to water sensitivity of clay is particularly important for UBD design. The primary means is to analysis the clay mineral content of the drilling section implemented UBD, and to evaluate the level in rocks according to the natural gamma ray logging data. The higher the clay content is, the stronger is the water sensitivity of the formation3. Especially, more attention should be pay to screening pure gas or foam drilling.

\section{Formation pore pressure and collapse pressure}

\subsection{Formation pore pressure}

Pre-drill prediction of pore pressure is used as input in the well planning phase and is based on an assumption of the local geology in the prospect, typically extrapolated from near-by wells. When clays are buried the overburden stress increases and the shale is compacted. This behaviour follows the Terzaghi (1929) stress relationship (Equation 1) between total stress (compressive stress developed by the sediment load),

\footnotetext{
* Corresponding author: zhuzhongxi@126.com
} 
effective stress (as can be seen as the sum of stresses acting on the grain to grain contacts), and the pore pressure.

$$
\sigma_{T}=\sigma+p_{P}
$$

Where $\sigma_{T}$ is the total stress, $\sigma$ is the effective stress and $p_{P}$ is the pore pressure. During deposition $\sigma_{T}$ will increase. When $\sigma_{T}$ is increased and at the same time the fluids are allowed to escape, $\sigma$ will increase while porepressure remains hydrostatic. The increase in $\sigma$ will compact the sediment and reduce its porosity.

Clay rich sediments with a uniform lithology will reduce porosity with depth as long as the fluids are allowed to escape. Numerous normal compaction curves versus effective stress or depth are given for different sedimentary basins and rock types (Rieke and Chillingarian, 1974; Baldwin and Butler, 1985). On the other hand if depositional rate is fast, the low permeability in clay rich sediments restricts the upward flow and creates overpressure (pressure above a normal hydrostatic gradient). This overpressure will hinder the compaction and stop the porosity reduction with depth trend. This trend deviation or stopped compaction can be used to estimate the amount of overpressure. Eaton (1975) proposed the Eaton ratio method to estimate overpressure on the form:

$$
p_{P}=\sigma_{T}-\left(\sigma_{T}-p_{H}\right) \times\left(\frac{C_{o}}{C_{N}}\right) c
$$

Where $p_{H}$ is the hydrostatic pore pressure, and $C_{o}$ is the observed attribute, and $C_{N}$ is the attribute when the pore pressure is hydrostatic, and $c$ is an empirical fitting constant for sonic compressional velocity data $(c=3)$ or restitivity data $(c=1.2)$. Since porosity is rarely measured directly, indirect measurements of porosity from logs such as acoustic velocity or restitivity data is used. The $C_{N}$ for the given depth of interest is not an actual measurement and the normal compaction trend has to be extrapolated for the depth interval where overpressure is encountered. The equivalent depth method also uses a reference normal compaction curve similar to Eaton's ratio method. Here the procedure is to compare the $C_{o}$ with the depth at which $C_{N}$ would be at the normal compaction curve and use this value to determine the pore pressure (Ham, H.H. 1966).

The accurate degree of mastering for formation pressure directly affects that whether the underbalanced state can be truly realized. Only accurately mastering the formation pore pressure, can the density of drilling fluid be effectively determined, can the bottom underpressured value be well designed and can the well control device be chosen. At present the calculation methods and models on formation pressure are more, this paper only select relatively simple models to explain problems. For the new exploration area, the formation pressure is usually obtained through seismic interval velocity or velocity spectral analysis by geological department. For the well with actual drilling data, the formation pressure is obtained through actual drilling fluid density, density and acoustic travel time logging and other data combining with abnormal high pressure uncompacted theory, the applicability of which method is well for sand-shale profile mainly in mudstone, the formula is shown in (1).

$$
V_{p}=a+k p_{e}-b e^{-d p_{e}}
$$

Where, $V_{p}$ is rock compressional velocity; $a, k, b, d$ are experience factors relevant to formation; $p_{e}$ is rock effective stress.

\subsection{Formation collapse pressure}

The drilling fluid annular equivalent density during implementing underbalanced drilling is lower than that of conventional drilling, if the equivalent density is lower than the formation collapse pressure equivalent density, sidewall collapse will occur, therefore, during the underbalanced drilling, the annulus pressure must be guaranteed not less than the collapse pressure (It is certain that the annulus pressure is not less than the collapse pressure). Usually formation collapse pressure is affected by mechanical and chemical two combined factors, without considering the influence of chemical factors, the elastic mechanic theory can be used to describe the rock stress state around sidewall after drilling. Any cross section of borehole can be regarded as a round hole of infinite plane, which generate stress concentration around the round hole, if the maximum shear stress suffered on the rock is more than its shear strength, shear failure will occur. By use of adjacent well logging, fracturing and drilling data, the corresponding equivalent mud density of the largest shear stress suffered on the sidewall rock can be calculated, that is, collapse pressure equivalent density:

For vertical wells there is

$$
\rho_{t}=\frac{2 v}{1-v} \rho_{e}
$$

For horizontal wells there is

$$
\rho_{t}=\frac{3-4 v}{1-v} \rho_{e}
$$

Where, $\rho_{f}$ is wall collapse equivalent density, $\mathrm{g} / \mathrm{cm}^{3} ; \rho_{e}$ is equivalent density of rock effective stress, $\mathrm{g} / \mathrm{cm}^{3} ; v$ is poisson's ratio of rock, dimensionless.

As the drilling fluid currently used is main in waterbased and logging is implemented after borehole drilling finished, this moment the sidewall has been soaked by the drilling fluid, the data has already been the result of chemical effects and stress effects of hydration between drilling fluid and sidewall. Studies have shown that, with the containing formation of water-based drilling fluid mud soaked in water after soaking intensity ratio did not decline in drilling [2], and with soaking time, pressure drop, the more extended collapse. The intensity of formation containing mud after soaked in water-based drilling fluid will decline compared with not soaked in water-based drilling fluid, and with the lengthening of soaking time, and the longer the soaking time is, the more the declination of pressure drop is. Thus, the factor should be fully considered in pure gas drilling, fog drilling, and foam drilling. 


\section{Situation of formation water oil and gas production}

Formation water production is an important factor that affects gas drilling, excessive formation water can bring difficulty to carrying cuttings for pure gas drilling, even not impossible to continue drilling, so the prediction of layer and size of formation water is an important indicator to the choice of drilling modes and the establishment of contingency plans for drilling method transition.

On the problem of judgment of water layer in the formation, the application of well logging data can well explain it. However, in under-balanced drilling, especially gas drilling, the size of formation water yield is the necessary condition of the decision of underbalanced drilling modes and how to implement drilling method transition in the upcoming drilling layer. Presently the related prediction theory for the size of formation water yield has been researched[4-5], and the correlative study is mainly from logging data interpretation, Percolation mechanics theory, statistical method of regional real drilling data. For the current study situation, the use of regional real drilling logging and formation testing or well testing information for statistics is a more practical approach, but for new regional exploration well, is not, yet need more deepgoing and meticulous research.

The ultimate goal of oil and gas drilling is to found oil and gas, currently most of the ground test data are carried out around the hydrocarbon zone. The forecasts for oil and gas production of formation usually reference to existing test data, but the worst case should be considered for new regional exploration well appropriately.

\section{Engineering feasibility of underbalanced drilling}

\subsection{Equipment of gas drilling}

The Evaluation of gas drilling equipment can be stared from two aspects, the first is the size of gas quantity required for gas drilling, the second is the size of injection pressure required for gas drilling. According to the required gas volume, the matching compressor and booster should be selected, if needed sets are too much even can not be met or placed by the constraint of field, other drilling modes should be considered. In the same borehole conditions for gas drilling, the needed gas of pure gas drilling is the most and the gas injection pressure is the least, while the aerated drilling oppositely.

\subsubsection{Related calculation of pure gas drilling}

In the construction of gas drilling, maintaining adequate gas flow is the key to drilling smoothly. If the gas flow is too low will lead to inadequate capacity to carrying cutting, and even lead to sticking, but too high will cause both devices, energy waste, and there are likely to reduce the the strength and stability of sidewall, casing and drilling tools by excessive erosion. Currently Angel model modified by Guo is commonly used for minimum gas flow calculation. Angel mode think that under the required atmospheric conditions, the minimum annular flow rate is $15.24 \mathrm{~m} / \mathrm{s}$, which can effectively carry solid particles in borehole. However, because of the borehole of gas drilling is relatively deep, the pressures and temperatures are different at different depths, so the speed under Angel standard condition should be converted into minimum kinetic energy form, that the minimum kinetic energy of per unit volume remain unchanged $\left(143.65 \mathrm{~J} / \mathrm{m}^{3}\right)$. Field practice comparison show that, the calculating gas quantity of Angel model modified by friction coefficient of Nikuradse introduced is $20 \%$ lower than that of the actual demand. Therefore, that should be taken into account in gas drilling.

\subsubsection{Related calculation of mist drilling and foam drilling}

Mist drilling as a supplement form to pure gas drilling, generally is to add some surfactant to the injected gas so as to inhibit the unity of water droplets and cuttings, when there is a small amount of water production in the formation, in order to prevent accumulation of cuttings in the annulus. Usually mist drilling is only used as transitional method between the pure gas drilling and foam drilling.

The biggest difference between foam drilling and pure gas drilling is that the former is more able to adapt to the formation water production situation, because the stronger ability to carry cuttings of foam itself, the required gas volume is smaller. However, when conducting foam drilling, it is ensure that all the annulus are in foam state, that is, the quality of the foam must be 0.55 to 0.96 range. Otherwise, less than 0.55 at the bottom there will be gas-liquid separation and slug flow appear, that its ability to carry cuttings rapidly decline; higher than 0.96 at the wellhead there will be annular flow pattern which lead to the cuttings sinking rapidly, carrying rock poorly and other issues. From the perspective of current research situation, the difficulties of foam drilling mainly is how to keep foam stable in wellbore, influencing factors are more and relevant calculation is relatively complicated. Usually the target of evaluating foam drilling is the volume of base fluid, as the foam base fluid should be defoaming and purification treatment when it reaches the ground to reuse, currently the research on reutilization of foambased fluid is developing in depth, and there is no detailed discussion here.

\subsubsection{Related calculation of aerated drilling}

Aerated drilling usually adopts conventional drilling fluid filled with gas to reduce the annulus equivalent density $\left(0.7 \sim 0.9 \mathrm{~g} / \mathrm{cm}^{3}\right)$ [3], which is a better drilling modes to deal with formation water production, well kick and lost circulation and other complex formation. The annulus fluid column pressure of aerated drilling is 
relatively high compared with that of foam drilling, usually the related calculations of gas-liquid two-phase flow is adopted, the required injection pressure is higher and the booster should be equipped with.

\subsection{Casing collapsing strength}

In the implementation of underbalanced drilling, the annular pressure is low, especially in the pure gas drilling, the borehole even can be regarded as completely emptying state. UBD design, especially for the conventional design which is completed, it must be considered that whether the collapse strength of upper casing is come up to the safety standard when implementing underbalanced drilling.

\subsection{Ground control equipment}

\subsubsection{The matching problem between upper casing and blowout preventer of under-balanced construction well section}

In general, the higher the pressure grade of BOP is, the smaller the largest type of the drift diameter is, such as the maximum drift diameter of currently used $35 \mathrm{MPa}$, $70 \mathrm{MPa}$ ram blowout preventer is $9346 \mathrm{~mm}$ (FZ35-35 or FZ35-70). If the situation is rather special, when the casing is $\varphi 508 \mathrm{~mm}$, and formation pressure is higher, well control demand that the pressure grade of wellhead BOP should reach $35 \mathrm{MPa}$, and that presently there is little BOP matching of the 9508 casing, this moment using underbalanced drilling will increase the risk of blow out of control.

\subsubsection{The problem of processing capacity of ground skimming tank, liquid-gas separator and blow off tank volume}

Drilling aerated drilling, if the excessive oil production exceed the processing capacity of ground skimming tank, or too much gas production exceed the processing capacity of liquid-gas separator, or too much formation water production exceed the blow off tank volume in foam drilling, it is important to ensure that the relevant preventive measures and contingency plans be done well.

\section{Application example}

A risk exploration wells of Xinjiang Oilfield is taken as an example for feasibility analysis. Design depth is $6350 \mathrm{~m}$ and the well structure is fourth opening. Because the drillability of Xiazijie formation, Fengcheng formation, and Jiamuhe formation of Permian in the third or fourth opening is poor, underbalanced drilling technology is Decided to use, which not only makes it possible to increase the penetration rate, but also can found and protect the reservoir. Therefore, feasibility analysis of underbalanced drilling is conducted respectively for three, four opening formation.According to the oil testing data of six drilled wells, the statistics of oil gas and water production conditions has been conducted for drilled $\mathrm{X}$ formation, $\mathrm{F}$ formation, and $\mathrm{J}$ formation and the statistical results are shown in Table 1.

Table 1 Well testing results - statistics of oil gas and water production on formation

\begin{tabular}{ccccc}
\hline Layer & $\begin{array}{c}\text { Thickness } \\
(\mathrm{m})\end{array}$ & $\begin{array}{c}\text { Oil } \\
\text { production } \\
(\mathrm{t} / \mathrm{d})\end{array}$ & $\begin{array}{c}\text { Gas } \\
\text { production } \\
\left(\times 10^{4} \mathrm{~m}^{3} / \mathrm{d}\right)\end{array}$ & $\begin{array}{c}\text { Water } \\
\text { production } \\
\left(\mathrm{m}^{3} / \mathrm{d}\right)\end{array}$ \\
\hline $\mathrm{X}$ & 28.56 & 49.33 & 0.12 & 8.46 \\
$\mathrm{~F}$ & 41.67 & 24.41 & 0.75 & 25.38 \\
\hline
\end{tabular}

Adopting the form of table for the calculation results, the comprehensive evaluation (shown in Table 2) is conducted by taking the weight of single index as the same. The drilling method should be selected on the basis of comprehensive evaluation results, the foam drilling for the third opening and the aerated water base drilling for the fourth drilling is a comparatively ideal selection.

Table 2 Comparison and comprehensive evaluation of various drilling modes

\begin{tabular}{ccc}
\hline $\begin{array}{c}\text { Opening } \\
\text { times }\end{array}$ & Drilling modes & $\begin{array}{c}\text { Comprehensive } \\
\text { evaluation }\end{array}$ \\
\hline \multirow{3}{*}{ Third } & Pure gas & 1A 3B2C \\
& Foam & 1A5B 0C \\
& Aerated & 4A1B 1C \\
Fourth & Pure gas & 1A3B 2C \\
& Foam & 0A 5B 1C \\
& Aerated & 5A 1B 0C \\
\hline
\end{tabular}

\section{References}

1. Huang Lindong, Wang Yongji, Li Ruiying, et al. Research On Some Problems In Underbalanced Drilling Design. Drilling \& Production Technology, 2002, 25(1): 7-10.

2. Saad E. Alajimi, Jerome J. Schubert. Optimum Selection Of Underbalanced Techniques. SPE/IADC 85322, 2003.

3. Yong Shihe, Zhang Chaomo. Logging Data Processing And Integrated Interpretation, Shandong Dongying: Petroleum University Press, 1996.

4. Huang Rongzun, Chen Mian, Deng Jingen, et al. Study On Shale Stability Wellbore By Mechanics Coupling With Chemistry Method[J]. Drilling Fluid \& Completion Fluid, 1995, 12(3): 15-21.

5. Zou Lingzhan, Deng Jingen, Wang Haige. Quantitative Prediction of Formation Water Production during Gas Drilling $[\mathrm{J}]$. Petroleum Drilling Techniques, , 2009, 37(3): 30-33. 